\title{
Durch Reflexion zum sprachbewussten Unterricht - Portfolio als ein Professionalisierungsinstrument für Deutsch als Zweitsprache
}

\author{
Magdalena Michalak \& Kirstin Ulrich (Erlangen-Nürnberg/Deutschland)
}

\section{Einleitung}

Lehrerprofessionalität lässt sich als Zusammenwirken von berufsbezogenem Wissen, situationsspezifischem Können sowie berufsethischen Haltungen beschreiben (vgl. Terhart 2007: 460). Ihre Entwicklung wird durch Reflexion angebahnt, durch die das eigene pädagogische Handeln analysiert, das Unterrichtsgeschehen evaluiert, mögliche Herausforderungen erkannt und Folgen abgeleitet werden (vgl. Neuweg 2005: 220, Häcker \& Winter 2011: 229, Roters 2012: 111). Setzt man voraus, dass die Reflexionsprozesse durch die schriftliche Ausarbeitung eigener Gedanken und den anschließenden Austausch eingeleitet und vertieft werden, kann die Reflexionsfähigkeit angehender Lehrkräfte durch ein Portfolio gezielt entfaltet werden. Zugleich wird das Portfolio zu einem Arbeitsinstrument, das eine starke Verknüpfung zwischen Theorie und Praxis bietet und die Aktivitäten der Lehramtsstudierenden unter einem bestimmten Aspekt steuert.

Im folgenden Beitrag wird für die reflexive Bearbeitung das pädagogische Handeln in mehrsprachigen Klassen in den Blick genommen. Hierfür wird zunächst die Lehrerprofessionalität aus der Perspektive des Deutschen als Zweitsprache (DaZ) beleuchtet. In einem nächsten Schritt wird anhand des LIDAG1-

\footnotetext{
${ }^{1}$ LIDAG - Linguistische und didaktische Grundlagen für den (Fach-)Unterricht mit Kindern und
} Jugendlichen nichtdeutscher Muttersprachen (zur Projektbeschreibung vgl. Michalak 2013, 2014). 
Portfolios gezeigt, wie die professionsbezogene Selbsterkundung und die Entwicklung eigener Lehrerprofessionalität für die sprachliche Vielfalt durch reflexives Schreiben und anschließende Beratungsgespräche im Rahmen einer praxisorientierten Ausbildung systematisch gestärkt werden können. Orientiert an den Deskriptoren für die Selbstbeurteilung und Reflexion werden die einzelnen Bausteine des LIDAG-Portfolios erläutert. Zudem wird ein Kriterienkatalog vorgestellt, der als Grundlage für ein Feedbackgespräch bzw. als ein Beurteilungsleitfaden der Portfolioarbeit dienen kann.

\section{Lehrerprofessionalität aus der Perspektive DaZ}

Die gesellschaftlichen Entwicklungen wirken sich auf die Anforderungen an die Lehrkräfte aller Fächer aus und stellen sie vor die Aufgabe, Schülerinnen und Schüler nicht nur fachlich, sondern zugleich auch (fach-)sprachlich bestmöglich zu unterstützen. Die Lehrkräfte sollten daher zum einen den Bildungsauftrag übernehmen, insbesondere den neu zugewanderten Schülerinnen und Schülern grundlegende Deutschkenntnisse zu vermitteln, die für eine erfolgreiche Teilhabe am Unterricht erforderlich sind. Zum anderen wird von den Lehrpersonen erwartet, die diversen sprachlichen Lernvoraussetzungen und Erfahrungen der Schülerinnen und Schüler in ihrem Unterricht angemessen zu berücksichtigen und differenzierte Lehrangebote zu entwickeln, mit denen alle Lernenden gleichermaßen gefördert werden können. Um der sprachlichen und kulturellen Vielfalt gerecht zu werden, benötigen die (künftigen) Lehrkräfte somit ein spezifisches theoretisches und praktisches Wissen über den Erwerb und die Vermittlung einer übergreifenden Sprachkompetenz hin zu fachlicher Kommunikation in deutscher Sprache. Dieses Wissen ermöglicht die Integration von Sprachförderung aller Schülerinnen und Schüler in jeden Unterricht.

Da die Lehrkräfte auf diese Aufgaben schon in ihrer Ausbildung effektiv vorbereitet werden sollten, werden hierfür bundesweit Angebote geschaffen, in denen jedoch die Gewichtung der DaZ-spezifischen Inhalte im Gesamtstudienangebot unterschiedlich festgelegt ist (vgl. Baumann 2017: 16). Neben Studiengängen, die obligatorisch im Rahmen von Grundlagenmodulen für Lehramtsstudierende aller Fächer und Schulformen im Bereich DaZ oder Sprachbildung wie in NordrheinWestfalen oder Berlin eingeführt wurden, gibt es auch Bundesländer wie Bayern oder Hamburg, in denen die DaZ-Inhalte lediglich fakultativ studiert werden können. So variieren auch die Modelle, die den Konzepten zugrunde liegen: von Sensibilisierung und Erwerb einer Basisqualifikation bis zur Möglichkeit einer vertieften Qualifikation im Bereich DaZ (vgl. Seipp \& Baumann 2015: 18f.). Ebenso unterschiedlich ist die Verankerung der Studienangebote: ausgehend von den einzelnen Fächern bis zur Andockung an die Germanistik. Die gleiche Uneinheitlichkeit gilt für den Umfang des Studiums: von Grundlagenmodulen im Umfang 
von fünf Leistungspunkten bis zu Unterrichtsfachstudiengängen mit 55 Leistungspunkten (vgl. Baumann 2017: 18-20).

Für die Qualifizierung von Fachlehrkräften in DaZ im schulischen Kontext liegen bislang keine Standards vor. Aktuelle Studienangebote in diesem Bereich orientieren sich an den KMK-Standards der Lehrerbildung, die zwar dem Umgang mit „Heterogenität und Vielfalt als Bedingungen von Schule und Unterricht“" eine besondere Bedeutung zuschreiben (KMK 2004: 5), auf die DaZ-spezifischen Lehrkompetenzen aber nicht explizit eingehen. Ausschließlich für Deutsch- und Fremdsprachenlehrende sehen die Fachprofile der KMK die Auseinandersetzung mit Theorien des Sprachlernens und individuellen Voraussetzungen des Spracherwerbs auch unter Berücksichtigung migrationsbedingter Mehrsprachigkeit und interkultureller Kontexte sowie mit Sprachvergleich im Studium als notwendig vor (vgl. KMK 2008: 28, 45). Betrachtet man Kompetenzmodellierungen für angehende Fremdsprachenlehrende, wird jedoch deutlich, dass sie sich auf die Besonderheiten der Arbeit im Fremdsprachenunterricht beziehen, der sowohl an die Lehrpersonen als auch an die Schülerinnen und Schüler andere Anforderungen als das Lehren und Lernen in der Zweitsprache Deutsch stellt (vgl. Newby et al. 2008).

Eine inhaltliche Strukturierung von DaZ-Lehrkompetenzen schlägt das Projekt DaZKom $^{2}$ vor (vgl. Köker et al. 2015). Das entwickelte Modell baut auf dem inhaltlichen Kern des Faches DaZ auf, das „die Bereiche Linguistik des Deutschen, Fremd- bzw. Zweitsprachenlehr- und -lernforschung sowie Didaktik und Methodik umfasst" (Köker et al. 2015: 183). Diese Themengebiete bilden die inhaltliche Grundlage der definierten DaZ-Kompetenzen in drei Dimensionen: (1) linguistisches Fachregister mit dem Fokus auf Sprache, (2) Mehrsprachigkeit mit dem Fokus auf den zweitsprachlichen Lernprozess und (3) Didaktik mit dem Fokus auf den Lehrprozess (vgl. Köker et al. 2015: 184). In dem Modell konzentriert sich das DaZ-spezifische Professionswissen und -können dementsprechend auf sprachwissenschaftliche und -didaktische Inhalte. Die Einstellungen, die u.a. Offenheit anderen Kulturen und Sprachen gegenüber, Flexibilität oder soziales Engagement und Reflexionsfähigkeit einbeziehen (vgl. EUCIM-TE 2011) und die in anderen allgemeinpädagogischen Kompetenzmodellen unterschiedlich aufgefasst werden (vgl. z.B. Baumert \& Kunter 2011, Beck et al. 2008), finden in dem genannten Modell keine Berücksichtigung.

Eine breitere Ausdifferenzierung der DaZ-spezifischen Lehrkompetenzen in diesem Sinne wurde im Projekt LIDAG unternommen (vgl. Michalak 2013, 2014). Die hierbei definierten Teilkompetenzen der Lehrerprofessionalisierung, auf denen das LIDAG-Portfolio basiert, umfassen die Sach- und Diagnosekompetenz, die didaktisch-methodische Kompetenz sowie die Sozial- und Selbstkompetenz, die nicht nur für den Deutschunterricht bzw. DaZ-Unterricht relevant sind, sondern fachübergreifend gelten (vgl. Abb. 1).

${ }^{2}$ DaZKom - Projekt „Professionelle Kompetenzen angehender LehrerInnen (Sek I) im Bereich Deutsch als Zweitsprache (DaZ)“ (vgl. Köker et al. 2015). 


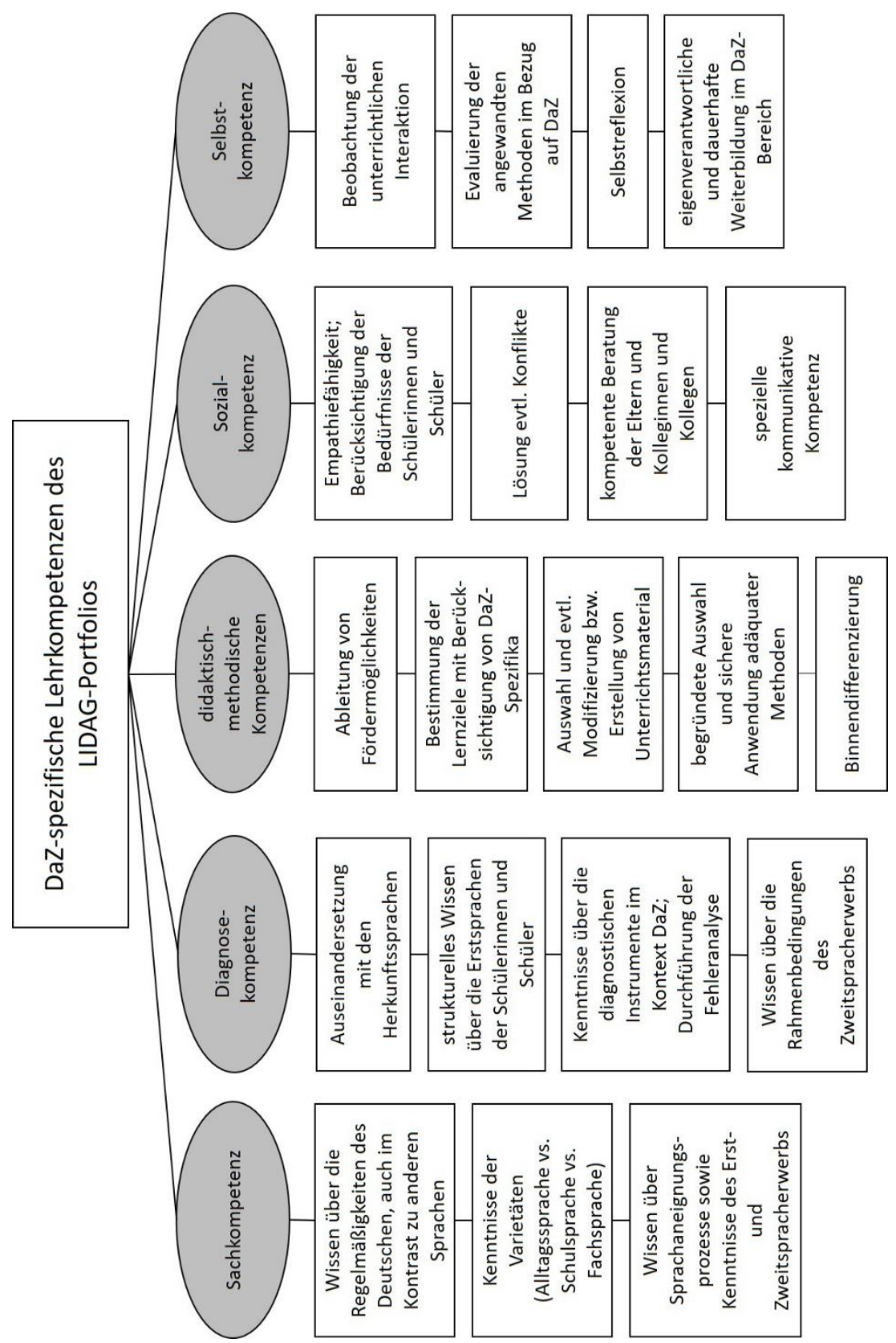

Abb. 1: Modellierung der DaZ-Kompetenzen im LIDAG-Portfolio

Unter Sachkompetenz wird das linguistische Grundlagenwissen verstanden, das der Lehrperson u.a. die Analyse von sprachlichen Anforderungen in ihrem Fach, Erfassung der sprachlichen Kenntnisse der Lernenden sowie die Auswahl passen- 
der sprachlicher Hilfestellungen ermöglicht. Neben Wissen über die Regularitäten des Deutschen sollten die Lehrkräfte die wichtigsten und schulrelevanten Register der deutschen Sprache - Alltags-, Bildungs- und Fachsprache - sowie ihre Charakteristika kennen. Dies bezieht auch die Kenntnisse verschiedener Darstellungsformen wie Diagramme, Tabellen, Schaubilder versus lineare Texte bzw. fachspezifische Textformen sowie sprachliche Handlungen ein, die durch die Denk- und Arbeitsweise des jeweiligen Fachbereichs geprägt sind. Sprachbewusster Unterricht setzt zudem eine Sensibilisierung für Sprachaneignungsprozesse unter dem Aspekt von Mehrsprachigkeit voraus, damit der Unterricht auf die mehrsprachigen Erfahrungen der Schülerinnen und Schüler gezielt aufbauen kann. Um ihre Lernpotenziale zu erkennen, eventuelle sprachliche Herausforderungen zu identifizieren und einen förderlichen Umgang mit Fehlern anzuregen, ist das Wissen über den Erstund Zweitspracherwerb unerlässlich.

Die Diagnosekompetenz umfasst die Fähigkeit, den Sprachstand einzuschätzen. Hierbei wird die Verknüpfung mit einer gut entwickelten Sachkompetenz deutlich: Die Kenntnisse der Phänomene der Lernersprachen sowie der Spracherwerbsphasen helfen, die sprachliche Kompetenz der Schülerinnen und Schüler zu diagnostizieren. Die Beschäftigung mit den Herkunftssprachen und der kontrastive Sprachenvergleich sind besonders wichtig für eine qualifizierte Fehleranalyse. Die Lehrkräfte sollten zudem passende Sprachstandserhebungsinstrumente aus dem Bereich DaZ kennen und sie gezielt anwenden können, um ausgehend von der Diagnostik den Unterricht sprachfördernd bzw. -bewusst zu planen. Des Weiteren sollten die Lehrpersonen außersprachliche Faktoren wie soziale, kognitive und kulturelle Voraussetzungen der Schülerinnen und Schüler in ihre Diagnose und Förderplanung einbeziehen.

Die DaZ-spezifische didaktisch-methodische Lehrkompetenz beinhaltet die Fähigkeit, bedarfsgerechte Lernziele zu bestimmen und den Unterricht dementsprechend sprachbewusst zu planen, durchzuführen und zu evaluieren. Die Lehrkräfte sollen demnach Lerninhalte, didaktische Ansätze und Methoden, Material sowie Medien nach DaZ-förderlichen Kriterien auswählen. Hierzu zählt die Fähigkeit, Materialien zielgruppengerecht zu modifizieren. Sprachförderliche Sozialformen bzw. adäquate Formen der Binnendifferenzierung bekommen dabei einen hohen Stellenwert.

Die Sozialkompetenz umfasst die Gesamtheit von Fähigkeiten und Einstellungen, die zur Verständigung beitragen. Hierzu zählen Toleranz gegenüber anderen Sprachen und Kulturen sowie Empathiefähigkeit bzw. die Bereitschaft, Empathie auszubilden. Sozialkompetente Lehrkräfte im Bereich DaZ können sich auf die besonderen Bedürfnisse ihrer Schülerinnen und Schüler einlassen. Sie können Spannungen abbauen und eventuelle Konflikte lösen. Wertschätzung und eine angemessene kommunikative Kompetenz helfen den Lehrkräften, den Unterricht orientiert an den Bedürfnissen der Lernenden zu gestalten. Damit sind z.B. die Reflexion über das angemessene Sprechtempo, die Feinsinnigkeit bei der Auswahl einer sprachlichen Varietät und bei der Fokussierung grammatischer Korrektheit, 
hinsichtlich quantitativ und qualitativ hochwertigen sprachlichen Inputs oder Sensibilität bei der Formulierung von Elternbriefen gemeint. Diese Fähigkeiten bilden die Grundlage für eine kompetente Beratung der Lernenden, ihrer Eltern sowie Kolleginnen und Kollegen u.a. in Bezug auf Spracherwerb und -vermittlung.

Die Selbstkompetenz legt den Fokus auf die Selbstreflexionsfähigkeit der Lehrkräfte. Diese umfasst die Bereitschaft, sich im Unterricht selbst zu beobachten und dabei kritisch zu hinterfragen. Die Lehrkräfte sollten in der Lage sein, sich fachrelevant über die Neuerungen auf politischer Ebene sowie im Bereich der Schulentwicklung bezogen auf DaZ zu informieren. Dies setzt die Bereitschaft voraus, sich stetig weiterzubilden und gegebenenfalls eigenen Unterricht im Einklang mit neuen Forschungserkenntnissen im Bereich DaZ neu auszurichten.

Die so definierten DaZ-spezifischen Lehrkompetenzen bilden den Ausgangspunkt für die Konzipierung des im Folgenden dargestellten LIDAG-Portfolios. Dabei gelten die beschriebenen (Teil-)Lehrkompetenzen als Deskriptoren für die kritische Selbstreflexion über die Entwicklung eigener Lehrerprofessionalität im Bereich DaZ.

\section{Das LIDAG-Portfolio}

Lehrerprofessionalität entwickelt sich in einem längeren Prozess. Die Basis hierfür wird in den Veranstaltungen in der ersten Phase der Lehrerbildung gelegt, in denen die Lehramtsstudierenden in erster Linie theoretische Grundlagen für praktisches Handeln erwerben. So belegen Studien, dass im Studium vermitteltes explizites Grammatikwissen die angemessene Auswertung einer Sprachstandsanalyse begünstigt. Gleichzeitig zeigt sich, dass dabei diejenigen Studierenden besser abschneiden, die bereits über praktische Erfahrungen im Bereich der Durchführung und Auswertung von Sprachstandsanalysen verfügen (vgl. Ulrich in Vorb.). Hier zeigt sich die Relevanz der Verknüpfung von Sachkompetenz und Diagnosekompetenz sowie der praktischen Erfahrung. Wird das Theoriewissen im Lehramtsstudium wenig anwendungsbezogen vermittelt, kann dies zur Entwicklung von trägem Wissen (vgl. Gruber \& Renkl 2000) führen, das Lehramtsstudierende nicht auf Situationen im schulischen Alltag anwenden können (vgl. Neuweg 2007). Um dem entgegenzuwirken, können Lehr-Lerninstrumente eingesetzt werden, die eine enge Theorie-Praxis-Verzahnung ermöglichen. So kann die Auseinandersetzung mit der DaZ-Spezifik durch die Verbindung von mehreren Komponenten und Praxiselementen wie das Mitwirken an Veranstaltungen, die Durchführung eines (Förder-) Unterrichts, Verfassen eines Portfolios und Beratungsgespräche intensiviert werden.

Da die Praktika im Lehramtsstudium in Bildungswissenschaften und unterschiedlichen Fächern absolviert werden, besteht nicht immer die Möglichkeit, den Fokus explizit auf die heterogene Schülerschaft zu lenken. Dies wird tendenziell in den „klassischen“ Praktikumsberichten deutlich, die meist keine bestimmte Lern- 
zielgruppe bzw. keinen bestimmten Lehr-Lern-Zugang fokussieren. Die Portfolioarbeit - durch das Schreiben als reflexive Praxis - ermöglicht den Studierenden hingegen, die Entwicklung eigener Lehrerprofessionalität längerfristig und eigenverantwortlich zu begleiten (vgl. Ballweg \& Bräuer 2011, Bräuer \& Schindler 2011). Damit wird im Lehramtsstudium das Ziel verfolgt, die Studierenden zu reflective practitioners (Burwitz-Melzer 2004: 144) auszubilden, die das erlernte Wissen mit dem analysierten Unterricht verknüpfen, die Lernfortschritte der Schülerschaft und der eigenen Lehrerpersönlichkeit einschätzen, organisieren und kontrollieren können (vgl. Burwitz-Melzer 2004: 144). Somit wird auch ein Hauptaugenmerk auf die Beobachtungsfähigkeit gerichtet. Da die Beobachtungen und Analysen verschriftlicht werden, kann jederzeit wieder nachgelesen werden, welchen Weg der Problemlösung die Studierenden gegangen sind. Die fixierten Gedanken können weiterentwickelt und überarbeitet werden. Die Studierenden denken schreibend über Tätigkeiten z.B. in den Unterrichtsbeobachtungen und -versuchen nach und setzen sich mit dem schriftlich Fixierten auseinander (vgl. Bräuer 2000). Damit werden neben der Fähigkeit zur Reflexion auch die Fähigkeit zur Anfertigung von lesewirksamen Texten und die Problemlösefähigkeit erweitert. Dadurch kann auch die/der Dozierende einen Einblick in die Arbeits- und Denkweise sowie das Verständnis der Studierenden erhalten und die getroffenen Entscheidungen besser nachvollziehen. Die kritische Distanz zum eigenen Handeln im Unterricht und zum eigenen Lernverhalten wird nicht nur durch die Selbstevaluation im Portfolio, sondern auch durch den Austausch mit anderen Kommilitoninnen und Kommilitonen sowie der Mentorin bzw. dem Mentor effizient verstärkt (vgl. Michalak 2013: 233). Die Fixierung der Gedanken in kohärenten Texten ermöglicht zugleich die Ausarbeitung großer und komplexer mentaler Zusammenhänge über einen längeren Zeitraum hinweg, in dem die Praktika absolviert werden.

Die genannten Merkmale können in Einklang mit Weinerts Prinzip der Förderung des selbstregulierenden Lernens gebracht werden (vgl. Weinert 1982), welches die Gestaltung und Regulation des eigenen Lernprozesses in den Fokus rückt.

\subsection{Das Konzept des Portfolios}

Mit dem LIDAG-Portfolio wird ein Instrument zur Verfügung gestellt, das den Aufbau der DaZ-spezifischen Lehrkompetenzen systematisiert dokumentiert und einen reflektierten Dialog mit sich selbst und anderen Beteiligten über die Entwicklung eigener Lehrerprofessionalisierung ermöglicht. In diesem Sinne ist das LIDAG-Portfolio als ein Entwicklungsportfolio konzipiert, in dem Lernergebnisse und Lernprozesse im Bereich DaZ und des sprachbewussten Unterrichts - gekoppelt an Seminare und den selbstständig durchgeführten Unterricht - erfasst werden. Ziel des Portfolios ist es, eine Brücke zwischen dem wissenschaftlich fundierten DaZ-spezifischen Wissen und der praktischen Anwendung in der Schule zu schlagen und dabei die sprachlichen Zugänge der Schülerinnen und Schüler gezielt zu analysieren. Damit hat das Konzept des Portfolios den Anspruch, den 
forschungsbasierten Anforderungen zu entsprechen (vgl. Kunter et. al 2011). Da kriteriengeleitete Selbstanalysen einen tieferen Grad der reflexiven Auseinandersetzung mit dem Thema und eine bessere, systematische Verknüpfung der neuen Informationen mit dem Vorwissen erlauben (vgl. Hübner et al. 2007), erfolgt die Reflexion in dem LIDAG-Portfolio kriteriengesteuert - angelehnt an die DaZspezifischen Lehrkompetenzen (vgl. Abb. 1). Anknüpfend an die Ergebnisse von Schäfer et al. (2012: 284f.), wird ein hochstrukturiertes Reflexionsformat gewählt, um die Anwendung tiefenorientierter Elaborationsstrategien stark zu fördern. Die Strukturierung der Reflexion mit dem LIDAG-Portfolio ist durch Leitfragen, Beobachtungsaufträge sowie andere Aufgabenstellungen (wie z.B. die Durchführung einer Bedarfsanalyse), grafische Elemente (wie z.B. die Darstellung eigener Sprachlernbiografie) und Ankreuzaufgaben (wie z.B. Einschätzung der Bedeutung der einzelnen DaZ-spezifischen Lehrkompetenzen für die eigene Arbeit) vorgegeben. Hierbei werden die Studierenden dazu ermutigt, nicht nur ihre Stärken und positiven Erfahrungen aufzuzeigen, sondern auch eigenes Entwicklungspotenzial zu analysieren oder negative Unterrichtserfahrungen zu reflektieren. Die strukturelle Anleitung wird ebenfalls zur Grundlage eines Dialogs über den Lernprozess (vgl. Klenowski 2002: 111) und bietet eine Hilfe für das Beratungsgespräch sowie für die Beurteilung, in die der Entwicklungsprozess einfließt (vgl. Ballweg \& Bräuer 2011: 10).

$\mathrm{Da}$ diese Vorgehensweise vor allem in der Anfangsphase einer intensiven, direkten Anleitung und Begleitung bedarf (vgl. Häcker \& Winter 2011: 228), sieht das didaktische Gesamtkonzept des LIDAG-Portfolios eine Einführung der Studierenden in seine Konzeption, in die Zielsetzung und die Handhabung des Reflexionsformats sowie in die Grundsätze der Portfolioarbeit vor. Dies schließt eine intensive Betreuung der Studierenden bei komplexeren Aufgaben des Portfolios wie die Durchführung einer Bedarfs- oder Fehleranalyse ein. Zusätzlich finden sich im gesamten Portfolio kurze Anleitungen, d.h. ein Manual, zum geeigneten Gebrauch der verschiedenen Elemente, in welchem jeweils auch kurz auf Ziele und Zwecke der Portfolioelemente eingegangen wird.

In seiner Ausgestaltung ist das LIDAG-Portfolio an das Europäische Portfolio für Sprachlehrende in Ausbildung - EPOSA angelehnt (vgl. Newby et al. 2008). Im Gegensatz zu EPOSA werden hier jedoch die eigenen Lehrkompetenzen nicht durch Kann-Beschreibungen evaluiert, sondern im Wesentlichen durch Leitfragen angeleitet. Da diese als Orientierung für die Selbstevaluation dienen, müssen nicht alle Leitfragen beantwortet werden. Dementsprechend ist das LIDAG-Portfolio so konstruiert, dass es genug Freiraum für die Studierenden bietet und für die jeweilige Arbeitssituation ergänzt bzw. modernisiert werden kann und muss. 
Tab. 1: Aufbau des LIDAG-Portfolios

\begin{tabular}{|c|c|l|}
\hline \multicolumn{2}{|c|}{ Inhalte des LIDAG-Portfolios } \\
\hline \multicolumn{2}{|c|}{ Angaben zur Person } & $\begin{array}{l}\text { - Name, Fächer, Semester usw. } \\
\text { - }\end{array}$ \\
\hline \multicolumn{2}{|c|}{ Ort des Förderunterrichts } \\
\hline
\end{tabular}

Das LIDAG-Portfolio besteht aus drei Hauptelementen (Tab. 1): Sprach- und Lernbiografie, Selbstbeurteilung und Dossier. Der erste Teil, der möglichst zu Beginn der Arbeit mit mehrsprachigen Klassen ausgefüllt werden sollte, setzt kein theoretisches Wissen voraus, sondern greift die Erfahrungen der Studierenden mit sprachlichen Varietäten sowie ihre eigenen Erfahrungen mit (Fremd-)Sprachen im Lern-/Lehrprozess auf. Systematisiert werden die Studierenden dazu angeleitet, ihre eigene Sprachlernbiografie darzustellen, eigene Mehrsprachigkeit zu erforschen und durch diesen Perspektivwechsel die Mehrsprachigkeit der Schülerinnen und Schüler bewusst wahrzunehmen. Durch weitere Arbeitsaufträge werden die Einstellungen, die Motivation und Erwartungen der Studierenden erfasst und hinterfragt.

Der zweite Teil - die Selbstbeurteilung eigener Lehrkompetenzen - bildet das Kernstück des Portfolios. Zunächst werden die im Rahmen des LIDAG-Projekts modellierten DaZ-spezifischen Lehrkompetenzen definiert, die den Ausgangs- 
punkt für die anschließende Selbstevaluation bilden. Im zweiten Schritt werden die Studierenden dazu angehalten, die Bedeutung der einzelnen Teilkompetenzen aus der DaZ-Perspektive einzuschätzen. Dieser Arbeitsauftrag dient explizit der Reflexion und zugleich implizit der Vertiefung in die Materie, da sich die Studierenden intensiv mit den notwendigen Kompetenzen befassen müssen. Anschließend werden die Studierenden über durchschnittlich acht Leitfragen zu je einer DaZrelevanten Lehrkompetenz zu einer wissenschaftlich fundierten Selbsteinschätzung angeleitet und auf diese Weise in der Entwicklung dieser Kompetenzen aktiv unterstützt.

Den dritten Baustein des LIDAG-Portfolios bildet das Dossier, das der Dokumentation und Sammlung von Unterrichtsmaterial, Nachweisen und Ergebnissen eigener inhaltlicher Arbeit dient. Hier werden beispielsweise Schülertexte, durchgeführte Bedarfsanalysen, Diagnoseergebnisse, Fehleranalysen, kurze Verlaufsprotokolle und handschriftlich gemachte Notizen zu durchgeführten Unterrichtseinheiten zusammengestellt. Für die einzelnen Arbeitsaufträge wird in dem Portfolio eine Anleitung angeboten. Durch die Auswahl der Dokumente werden zum einen die Unterrichtsplanung, die Vorgehensweise im Unterricht und zugleich eigene Fortschritte sichtbar gemacht. Zu diesem Zweck wird das Dossier beständig erweitert und verändert. Zum anderen bildet es die Grundlage für die eigentliche Selbstreflexion im zweiten Teil des LIDAG-Portfolios. Hierbei fungiert die Materialiensammlung auch wie ein Notizbuch, in dem insbesondere die Eindrücke direkt nach der durchgeführten oder beobachteten Unterrichtseinheit festgehalten werden. Da das Portfolio im Lehramtsstudium den Ansprüchen einer wissenschaftlichen Arbeit genügen sollte, werden die Studierenden dazu angehalten, die Beobachtungen, Entwürfe etc. mit Theoriekenntnissen und Literaturverweisen zu belegen. Dieser Anspruch bezieht nicht nur die Literatur- und Quellenarbeit, sondern auch die Zitierweise und die Auswahl des Sprachregisters ein.

\subsection{Auswertung der Portfolioarbeit}

Die Zielgruppe des LIDAG-Portfolios setzt sich zwar aus Personen aller drei Phasen der Lehrerbildung zusammen. Das Instrument wurde jedoch bisher immer analog zur Prüfungsform der Hausarbeit eingesetzt und damit musste es mit einem Leistungsnachweis bewertet werden. Dies kann kritisch betrachtet werden: Ausgehend von der aktuellen Debatte um die Benotung von Portfolios im Lehramtsstudium ist nämlich zu beachten, dass Portfolios immer einen reflexiven und damit einen sehr individuellen Teil enthalten und aus dem Grund nicht benotet werden sollten (vgl. Winter 2011, Ballweg \& Bräuer 2011, Richter 2011). Dagegen könnte argumentiert werden, dass ein Portfolio die Arbeit und Leistung des Lernenden bzw. Studierenden aufzeigen soll (vgl. Imhof \& Picard 2006). Es bleibt die Gefahr, dass das Portfolio aus dem Streben heraus angefertigt wird, den Dozierenden zu gefallen; der Notendruck kann sich auch negativ auf die Portfolioarbeit auswirken, da missglückte Unterrichtsmaterialien und -versuche bewusst weggelassen werden 
könnten. Bräuer (2011) spricht hier vom Widerspruch zwischen dem prozessorientierten Charakter der Portfolios und dem ergebnisorientierten Bildungssystem und schlägt Feedbackgespräche vor (vgl. Bräuer 2007).

Tab. 2: Beurteilungsraster des LIDAG-Portfolios

\begin{tabular}{|c|l|}
\hline & $\begin{array}{l}\text { Ist die Unterrichtsbeobachtung, -planung und -durchführung an den } \\
\text { Bedürfnissen der DaZ-Schülerinnen und Schüler orientiert? Sind die } \\
\text { Lernziele, die gewählten Methoden, Übungs- und Sozialformen sowie die } \\
\text { Materialien zielgruppengerecht? } \\
\text { Steht in jeder Phase die DaZ-Perspektive im Vordergrund? } \\
\text { Wurde handlungsorientiert und schüleraktivierend gearbeitet und die } \\
\text { Vermittlung von Strategien (Lernen lernen) eingeplant? } \\
\text { Wurden alle Sprachfertigkeiten in ausgeglichener Weise trainiert? }\end{array}$ \\
\hline Ist der Unterrichtsverlauf stringent, d.h. sind die einzelnen Elemente \\
aufeinander abgestimmt? \\
Ist der Unterricht prozess- oder produktorientiert?
\end{tabular}




Analysieren/Interpretieren
Über welches Wissen im Bereich DaZ verfügt die Lehrkraft und inwiefern
bezieht sie sich darauf?
Betrachtet sie selbstkritisch ihre DaZ-spezifischen Lehrkompetenzen?
Stellt sie eine Verbindung her zwischen ihrem Ausgangswissen im Bereich
DaZ und der Erfahrung während der Arbeit als Förderlehrkraft?
Bewerten/Beurteilen
Formuliert die/der Studierende eigene Stärken und Entwicklungspotentiale?
Nennt sie/er Ursachen?
Wie schätzt die Lehrkraft ihre Entwicklung DaZ-spezifischer
Lehrkompetenzen ein? Wie differenziert nimmt sie Bezug auf die einzelnen
Teilkompetenzen?
Wie kommt sie zu dieser Einschätzung und inwiefern hat die Portfolioarbeit
etwas damit zu tun bzw. etwas daran verändert?
Inwiefern arbeitet die/der Lehramtsstudierende nach eigener Einschätzung
sprachsensibler als zu Beginn der Portfolioarbeit?
Planen
Zieht die Lehrkraft Konsequenzen aus ihrem Kenntnisgewinn und formuliert
sie Lernziele für sich?
Was nimmt sich die/der Studierende konkret vor, um an den
Entwicklungspotenzialen zu arbeiten? Plant sie/er die Weiterentwicklung
einzelner Lehrkompetenzen im Bereich DaZ? Wie differenziert und präzise ist
sie/er hierbei?

Aufgrund der verschiedenen Positionen in der Wissenschaft wird für das LIDAGPortfolio ein zweistufiges Beurteilungssystem vorgeschlagen. Die Portfolios werden zunächst mithilfe eines Beurteilungsrasters (Tab. 2) durch die/den Dozentin/Dozenten evaluiert. Anschließend erhalten die Studierenden in einem strukturierten Feedbackgespräch eine detaillierte Rückmeldung zu ihren Leistungen. Der Kriterienkatalog (Tab. 2) hilft den Beraterinnen und Beratern, die Einschätzung der Reflexionsleistung der Lehramtsstudierenden zu strukturieren und sie gezielt zu beraten. Damit steht nicht die Benotung des Portfolios im Vordergrund des Beratungsgespräches, sondern die Frage, inwieweit die Portfolioarbeit zur persönlichen Entwicklung beigetragen hat und welche Strategien die Studierenden hieraus für ihr Studium und ihre Lehrtätigkeit in mehrsprachigen Klassen ableiten können. Dies setzt jedoch voraus, dass die Beurteilungsgesichtspunkte zu Beginn der Portfolioarbeit den Studierenden transparent gemacht werden.

Bei der kriteriengeleiteten Beurteilung des LIDAG-Portfolios werden formale und inhaltliche Teilbereiche berücksichtigt, die jedoch unterschiedlich gewichtet werden. Das Einhalten der formalen Standards (Vollständigkeit, angewandte Sprache und Gestaltung des Portfolios) fließt am geringsten in die eingebrachte Leistung ein. Der zweitwichtigste Bereich ist die Dokumentation, wohingegen der 
Reflexion über die DaZ-spezifischen Lehrkompetenzen eine zentrale Bedeutung zugeschrieben wird. Für die Einschätzung der professionellen Entwicklung der Studierenden wird das reflexive Schreiben den ,vier Ebenen der reflexiven Praxis“ zugeordnet: Dokumentieren/Beschreiben - Analysieren/Interpretieren - Bewerten/Beurteilen - Planen (vgl. Ballweg \& Bräuer 2011: 8). Auf der ersten Ebene werden DaZ-spezifische Elemente unkommentiert beschrieben. Der Unterrichtsverlauf und die eigene Vorgehensweise werden lediglich dokumentiert. Auf der komplexeren Ebene des Analysierens und Interpretierens werden die gemachten Erfahrungen konzeptualisiert, indem das beschriebene Wissen in den Kontext der Praxiserfahrung selbstkritisch eingeordnet und interpretiert wird (vgl. Ballweg \& Bräuer 2011: 8). Auf der dritten Entwicklungsstufe, der Ebene des Bewertens und Beurteilens, werden Arbeitsprodukte (Gesammeltes und Gelerntes) und Vorgaben (Kompetenzdefinitionen, Leitfragen) miteinander abgeglichen, um so zu einer Einschätzung der eigenen Leistung zu gelangen. Hier geht es also um die Fokussierung der eigenen Entwicklung, das Erkennen eigener Stärken und Schwächen. Auf der höchsten Ebene der reflexiven Schreibfähigkeit, auf der Ebene des Planens, „schließt sich die Reflexionsschleife auf einem höheren Erkenntnisstand“ (Ballweg \& Bräuer 2011: 8). Aus der Beurteilung (Stufe 3) werden die Konsequenzen gezogen: Die angehende Lehrkraft nimmt die gewonnenen Einsichten und die Selbstbeurteilung zum Anlass, um eigene Lernziele für ihre eigene Weiterarbeit zu formulieren, analog zu den Entwicklungspotentialen, die sie selbst erkannt und beschrieben hat. Das vierstufige Modell des reflexiven Schreibens wurde auf jede der fünf verschiedenen, im LIDAG-Projekt definierten DaZ-spezifischen Lehrkompetenzen (s. Kap. 2) bezogen. Die in der Tabelle 3 zusammengestellten, beispielhaften Ankerbeispiele beziehen sich ausschließlich auf die explizite Reflexion der Studierenden hinsichtlich der Sachkompetenz in einem Portfolio.

Nicht nur die eigene Praxis, sondern auch die wissenschaftlichen Theorien und die empirischen Belege, die das Portfolio beinhalten, sind der Ausgangspunkt für die persönlichen Entwicklungsgespräche und dienen zur Herausarbeitung der zukünftigen Entwicklung der Studierenden. Im Gespräch werden im Hinblick auf die eigenen Lernzuwächse Stärken und Schwächen der Studierenden diskutiert. Die Kommunikation über diese Aspekte wird damit auch zum Teil der Portfolioarbeit. Da das Portfolio meist mit einem Leistungsnachweis abgeschlossen wird, haben die Studierenden so die Möglichkeit, über die Note im Gespräch mitzubestimmen. Das LIDAG-Portfolio schlägt daher folgende Gewichtungsgrundlage für eine Benotung vor: 50 Prozent für die (schriftliche und mündliche) Reflexion, 40 Prozent für die Dokumentation und zehn Prozent für die formalen Aspekte. 
Tabelle 3: Ankerbeispiele aus den Portfolios der Studierenden

\begin{tabular}{|c|c|c|c|}
\hline 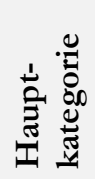 & $\begin{array}{l}\text { Allgemeine } \\
\text { Beschreibung }\end{array}$ & $\begin{array}{l}\text { Beschreibung der } \\
\text { Ausprägung auf } \\
\text { vier Ebenen der } \\
\text { Reflexion }\end{array}$ & $\begin{array}{l}\text { Ankerbeispiele aus den } \\
\text { Portfolios der Studierenden }\end{array}$ \\
\hline & $\begin{array}{l}\text { Das bloße } \\
\text { Sachverständnis im } \\
\text { Bereich DaZ, d.h. } \\
\text { das Wissen über } \\
\text { Regelmäßigkeiten } \\
\text { des Deutschen - } \\
\text { auch im Kontrast zu } \\
\text { anderen Sprachen - } \\
\text { sowie Kenntnisse } \\
\text { über Varietäten des } \\
\text { Deutschen und } \\
\text { Wissen über } \\
\text { Sprachaneignungs- } \\
\text { prozesse und den } \\
\text { Erst- und } \\
\text { Zweitspracherwerb }\end{array}$ & $\begin{array}{l}\text { Unkommentiertes } \\
\text { Beschreiben des } \\
\text { eigenen Wissens } \\
\text { über die deutsche } \\
\text { Sprache und der } \\
\text { Fachkenntnisse im } \\
\text { Bereich DaZ }\end{array}$ & $\begin{array}{l}\text { „Diese unangemessene } \\
\text { Verwendung des Registers, } \\
\text { der sich bereits eher an der } \\
\text { konzeptionell schriftlichen } \\
\text { Sprache orientiert, lässt sich } \\
\text { dadurch erklären, dass die } \\
\text { Schüler hauptsächlich in der } \\
\text { Schule Deutsch lernen. Hier } \\
\text { herrscht eine konzeptionell } \\
\text { schriftliche Sprache vor. Die } \\
\text { Schüler müssen lernen, in } \\
\text { welchen Kontexten welche } \\
\text { Register eingesetzt werden } \\
\text { sollten (Michalak 2008: 9) } \\
\text { d.h. in diesem konkreten Fall, } \\
\text { wann eine konzeptionell eher } \\
\text { schriftliche und wann die } \\
\text { mündliche Umgangssprache } \\
\text { angemessen ist.“ (SHMA: 11) }\end{array}$ \\
\hline 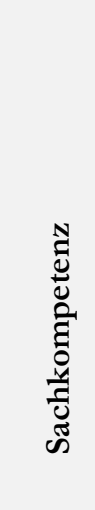 & & $\begin{array}{l}\text { Analyse und erstes } \\
\text { selbstkritisches } \\
\text { Einordnen des oben } \\
\text { beschriebenen } \\
\text { Wissens in den } \\
\text { Kontext der } \\
\text { Praxiserfahrung } \\
\text { (Kontextualisierung) }\end{array}$ & $\begin{array}{l}\text { „Erst im Nachhinein habe } \\
\text { ich gelernt, dass den } \\
\text { Erwerbssequenzen folgend, } \\
\text { die Stellung von nichtfiniten } \\
\text { Verbteilen erst nach dem } \\
\text { Erwerb von Nebensatz- } \\
\text { Strukturen erworben wird } \\
\text { [...]. Dementsprechend hätte } \\
\text { meine Reihenfolge - zuerst } \\
\text { trennbare Verben, dann } \\
\text { Nebensatzstrukturen - genau } \\
\text { andersherum sein müssen.“ } \\
\text { (REAR: 10) }\end{array}$ \\
\hline
\end{tabular}




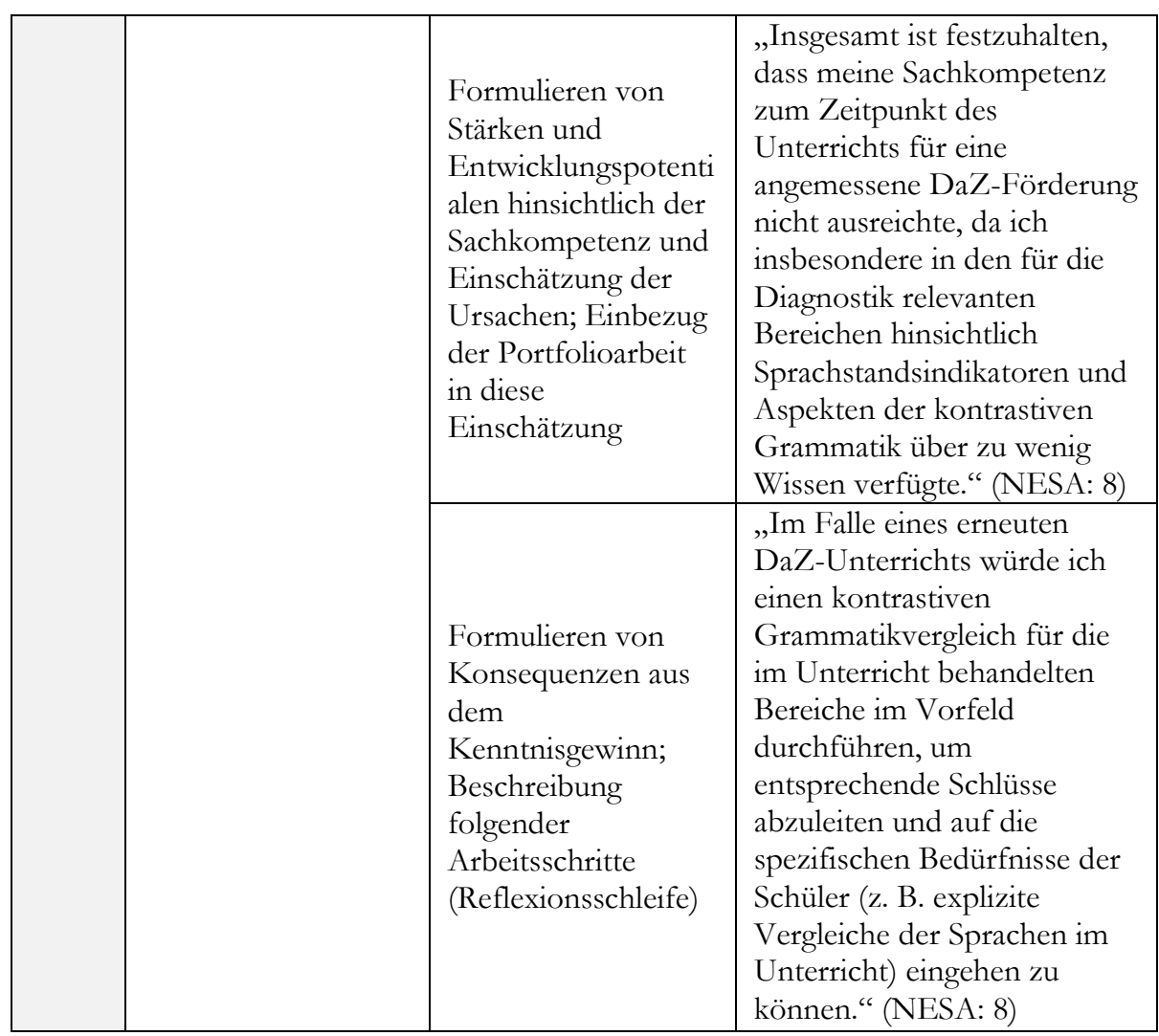

\section{$4 \quad$ Fazit}

In der aktuellen Debatte um die Lehrerprofessionalisierung wird die Dringlichkeit der Fokussierung auf die Sensibilisierung für die heterogene Schülerschaft in allen Schularten diskutiert. Diese Schülerschaft stellt die Lehrkräfte immer wieder vor neue Herausforderungen. Dabei geht es nicht ausschließlich um die sprachliche Förderung der Schülerschaft, sondern um die Verknüpfung des sprachlichen und fachlichen Lernens. Um dies umsetzen zu können, bedarf es einer gezielten Vorbereitung der angehenden und bereits praktizierenden Lehrkräfte auf den Umgang mit sprachlicher und kultureller Heterogenität. Dies kann u.a. durch das LIDAGPortfolio und das Beurteilungsraster erreicht werden. Die formative Evaluation des Reflexionsinstrumentes ergab, dass die Arbeit mit dem LIDAG-Portfolio einen hohen Nutzen hat. Das Portfolio unterstützt die Studierenden darin, ihre persönliche Entwicklung auf dem Weg zum Lehrberuf bewusst wahrzunehmen und dabei ihre persönlichen Stärken und Schwächen sowie ihre Ziele zu reflektieren. Insbesondere wurde das reflexive Schreiben als sehr gewinnbringend eingeschätzt, wie das folgende Beispiel aus dem Portfolio eines Studierenden belegt: 
Das Portfolio hat sich sehr bewährt, um sich Gedanken über sein eigenes Tun und Handeln $\mathrm{zu}$ machen. Dadurch, dass man alles genau aufschreibt, wird die Selbstreflexion angeregt. Während ich geschrieben habe, sind mir noch viele weitere Sachverhalte und Ideen in den Sinn gekommen, die mir wohl sonst nicht aufgefallen wären. [...] Durch die Fragen ist mir in vielen Fällen erst bewusstgeworden, welche Fähigkeiten beim Lehrerdasein eine Rolle spielen. [...] Das Portfolio hat dazu geführt, dass ich mich selbst ordnen konnte. Das heißt, ich habe in dem Berg von neuem Wissen genau reflektiert, welche Bereiche mir gut liegen und welche ich noch weiter ausbauen sollte. Dabei beziehe ich mich nicht nur auf DaZ, sondern auch auf die allgemeinen Lehrkompetenzen, wie Selbstoder Sozialkompetenzen (AVAS: 24).

Durch die Zusammenführung der Seminare zu DaZ-relevanten Aspekten, der Praxiserfahrung und der Arbeit mit dem Portfolio erzielen die Studierenden nicht nur einen Zuwachs in der Praxis im Umgang mit der heterogenen Schülerschaft, sondern erlangen eine höhere Reflexionsfähigkeit in Verbindung mit einer positiven Entwicklung der genannten DaZ-spezifischen Kompetenzen (vgl. Michalak 2014: 317). Die Portfolioarbeit ermöglicht einen strukturierten Perspektivwechsel von der Schüler- in die Lehrerrolle. Es bietet die Möglichkeit, das Berufsfeld systematisch zu erkunden und sensibilisiert für die Sprachlernerfahrung der Schülerinnen und Schüler sowie für die sprachbewusste Unterrichtsgestaltung. Gleichzeitig kann mit dem LIDAG-Portfolio eine andere Form der Prüfung im Lehramtsstudium herangezogen werden.

\section{Literatur}

Ballweg, Sandra; Bräuer, Gerd (2011): Portfolioarbeit im Fremdsprachenunterricht. Yes, we can! In: Fremdsprache Deutsch 45, 3-11.

Baumann, Barbara (2017): Sprachförderung und Deutsch als Zweitsprache in der Lehrerbildung - ein deutschlandweiter Überblick. In: Becker-Mrotzek, Michael; Rosenberg, Peter; Schroeder, Christoph; Witte, Annika (Hrsg.):

Deutsch als Zweitsprache in der Lehrerbildung. Münster, New York: Waxmann, 9-26.

Baumert, Jürgen; Kunter, Mareike (2011): Das Kompetenzmodell von COACTIV. In: Kunter, Mareike; Baumert, Jürgen; Blum, Werner; Klusmann, Uta; Krauss, Stefan; Neubrand, Michael (Hrsg.): Professionelle Kompetenz von Lehrkräften. Ergebnisse des Forschungsprogramms COACTIV. Münster, München: Waxmann, 29-53. 
Beck, Erwin; Baer, Matthias; Guldimann, Titus; Bischoff, Sonja; Brühwiler, Christian; Müller, Peter; Niedermann, Ruth; Rogolla, Marion; Vogt, Franziska (2008): Adaptive Lebrkompetenz. Analyse und Struktur, Veränderbarkeit und Wirkung handlungssteuernden Lehrerwissens. Münster: Waxmann.

Bräuer, Gerd (2000): Schreiben als reflexive Praxis. Freiburg im Breisgau: Fillibach.

Bräuer, Gerd (2007): Portfolios in der Lehrerausbildung als Grundlage für eine neue Lernkultur in der Schule. In: Gläser-Zikuda, Michaela (Hrsg.): Lemprozesse dokumentieren, reflektieren und beurteilen. Lerntagebuch und Portfolio in Bildungsforschung und Bildungspraxis. Bad Heilbrunn: Klinkhardt, 45-62.

Bräuer, Gerd (2011): Keine verordneten Hochglanz-Portfolios, bitte! Die Korruption einer schönen Idee? In: Brunner, Ilse; Häcker, Thomas; Winter, Felix (Hrsg.): Das Handbuch Portfolioarbeit. Konzepte, Anregungen, Erfahrungen aus Schule und Lehrerbildung. Seelze: Klett Kallmeyer, 257-260.

Bräuer, Gerd; Schindler, Kirsten (Hrsg.) (2011): Schreibarrangements für Schule, Hochschule, Beruf. Freiburg im Breisgau: Fillibach.

Burwitz-Melzer, Eva (2004): Das Lehramtsportfolio für Fremdsprachenlehrkräfte (LAPF). Auf dem Weg zum ,,reflective practitioner“. In: Zeitschrift für Fremdsprachenforschung 15/1, 143-157.

EUCIM-TE Consortium (Hrsg.) (2011): European Core Curriculum for Inclusive Academic Language Teaching (LALT): An Instrument for Training Pre-and In-service Teachers and Educators. Köln: Universität Köln.

Gruber, Hans; Renkl, Alexander (2000): Die Kluft zwischen Wissen und Handeln: Das Problem des trägen Wissens. In: Neuweg, Georg Hans (Hrsg.): Wissen Können - Reflexion: Ausgewählte Verhältnisbestimmungen. Innsbruck: Studien, 155-174.

Häcker, Thomas; Winter, Felix (2011): Portfolio - nicht um jeden Preis! Bedingungen und Voraussetzungen der Portfolioarbeit in der Lehrerausbildung. In: Brunner, Ilse; Häcker, Thomas; Winter, Felix (Hrsg.): Das Handbuch Portfolioarbeit. Konzepte, Anregungen, Erfahrungen aus Schule und Lehrerbildung. Seelze: Klett Kallmeyer, 227-233.

Hübner, Sandra; Nückles, Matthias; Renkl, Alexander (2007): Lerntagebücher als Medium des selbstgesteuerten Lernens - Wie viel instruktionale Unterstützung ist sinnvoll? In: Empirische Pädagogik 21/2, 119-137.

Imhof, Margarete; Picard, Christin (2006): Portfolio als Lern- und Bewertungsform in der Lehrerausbildung? In: Hilligus, Annegret Helen; Rinkens, Hans-Dieter (Hrsg.): Standards und Kompetenzen - neue Qualität in der Lebrerausbildung? Neue Ansätze und Erfahrungen in nationaler und internationaler Perspektive. Berlin: LIT, 259-265.

Klenowski, Val (2002): Developing Portfolios for Learning and Aassessment: Processes and Principles. London: Routledge Falmer. 
Köker, Anne; Rosenbrock-Agyei, Sonja; Ohm, Udo; Carlson, Sonja A.; Ehmke, Timo; Hammer, Svenja; Koch-Priewe, Barbara; Schulze, Nina (2015): DaZKom - Ein Modell von Lehrerkompetenz im Bereich Deutsch als Zweitsprache. In: Koch-Priewe, Barbara; Köker, Anne; Seifried, Jürgen; Wuttke, Eveline (Hrsg.): Kompetenzerwerb an Hochschulen: Modellierung und Messung. Zur Professionalisierung angehender Lehrerinnen und Lehrer sowie frühpädagogischer Fachkräfte. Bad Heilbrunn: Verlag Julius Klinkhardt, 177-205.

Kunter, Mareike; Kleikmann, Thilo; Klusmann, Uta; Richter, Dirk (2011): Die Entwicklung professioneller Kompetenz von Lehrkräften. In: Kunter, Mareike; Baumert, Jürgen; Blum, Werner; Klusmann, Uta; Krauss, Stefan; Neubrand, Michael (Hrsg.): Professionelle Kompetenz von Lebrkeäften. Ergebnisse des Forschungsprogramms COACTIV. Münster, München: Waxmann, 55-68.

Michalak, Magdalena (2013): Entwicklung von Lehrkompetenz im Kontext multikultureller Klassen. In: Vetter, Eva (Hrsg.): Professionalisierung für sprachliche Vielfalt. Perspektiven für eine neue Lehrerinnenbildung. Baltmannsweiler: Schneider Hohengehren, 210-237.

Michalak, Magdalena (2014): Das Lehrportfolio als Instrument professioneller Entwicklung im DaZ-Bereich. In: Bredel, Ursula; Ezhova-Heer, Irina; Schlickau, Stephan (Hrsg.): Zur Sprache.kom. Deutsch als Fremd-und Zweitsprache. Göttingen: Universitätsverlag, 301-321.

Neuweg, Georg Hans (2005): Emergenzbedingungen pädagogischer Könnerschaft. In: Heid, Helmut; Harteis, Christian (Hrsg.): Verwertbarkeit. Ein Qualitätskriterium (erziehungs-)wissenschaftlichen Wissens? Wiesbaden: VS Verlag für Sozialwissenschaften, 205-228.

Neuweg, Georg Hans (2007): Wie grau ist alle Theorie, wie grün des Lebens goldener Baum? LehrerInnenbildung im Spannungsfeld von Theorie und Praxis. In: Berufs- und Wirtschaftspädagogik - online 12, 1-14. http:/ /www. bwpat.de/ausgabe12/neuweg_bwpat12.pdf [01.03.2019].

Newby, David; Allan, Rebecca; Fenner, Anne-Brit; Jones, Barry; Komorowska, Hanna; Soghikyan, Kristine (2008): Europäisches Portfolio für Sprachlehrende in Ausbildung (EPOS A). Ein Instrument zur Reflexion. Europäisches Fremdsprachenzentrum des Europarates. http://archive.ecml.at/mtp2/publications/c3_ epostl_d_internet.pdf [01.03.2019].

Richter, Annette (2011): Portfolios im universitären Kontext: wann, wo, wie? Eine andere Bewertungsgrundlage im Seminarraum. In: Brunner, Ilse; Häcker, Thomas; Winter, Felix (Hrsg.): Das Handbuch Portfolioarbeit. Konzepte, Anregungen, Erfahrungen aus Schule und Lehrerbildung. Seelze: Klett Kallmeyer, 234-240.

Roters, Bianca (2012): Professionalisierung durch Reflexion in der Lehrerbildung. Eine empirische Studie an einer deutschen und einer US-amerikanischen Universität. Münster, München: Waxmann. 
Schäfer, Stefanie; Blomberg, Geraldine A.; Stürmer, Kathleen; Seidel, Tina (2012):

Der Einsatz von Lerntagebüchern in der universitären Lehrerausbildung Welchen Effekt haben strukturierende Leitfragen auf die Reflexionsfähigkeit von Lehramtsstudierenden? In: Empirische Pädagogik 26/2, 271-291.

Seipp, Bettina; Baumann, Barbara (2015): Deutsch als Zweitsprache in der Lehrerbildung - Spagat zwischen Hoffnung und Realität. In: Michalak, Magdalena; Kuchenreuther, Michaela (Hrsg.): Grundlagen der Sprachdidaktik Deutsch als Zweitsprache. Baltmannsweiler: Schneider Hohengehren, 8-33.

Ständige Konferenz der Kultusminister der Länder in der Bundesrepublik Deutschland (KMK), Sekretariat (2004): Standards für die Lehrerbildung. Bildungswissenschaft. Beschluss der Kultusministerkonferenz vom 16.12.2004. https:/ / www.kmk.org/fileadmin/Dateien/veroeffentlichungen_beschluesse/2004/ 2004_12_16-Standards-Lehrerbildung.pdf [01.03.2019].

Ständige Konferenz der Kultusminister der Länder in der Bundesrepublik Deutschland (KMK), Sekretariat (2008): Ländergemeinsame inhaltliche Anforderungen für die Fachwissenschaften und Fachdidaktiken in der Lehrerbildung. Beschluss der Kulturministerkonferenz vom 16.10.2008. https:/ /www.kmk.org/ fileadmin/Dateien/veroeffentlichungen_beschluesse/2008/2008_10_16 Fachprofile-Lehrerbildung.pdf [26.06.2019].

Terhart, Ewald (2007): Übersichtsartikel „Lehrer“. In: Tenorth, Heinz-Elmar; Tippelt, Rudolf (Hrsg.): Lexikon Pädagogik. Weinheim: Beltz, 458-461.

Ulrich, Kirstin (in Vorb.): Diagnostische Kompetenz in den Studiengängen für Deutsch als Zweit- (DaZ) und Fremdsprache (DaF).

Weinert, Franz Emanuel (1982): Selbstgesteuertes Lernen als Voraussetzung, Methode und Ziel des Unterrichts. In: Unterrichtswissenschaft 10/2, 99-110.

Winter, Felix (2011): Etwas, worauf man stolz sein kann. In: Brunner, Ilse; Häcker, Thomas; Winter, Felix (Hrsg.): Das Handbuch Portfolioarbeit. Konzepte, Anregungen, Erfahrungen aus Schule und Lehrerbildung. Seelze: Klett Kallmeyer, 19-26. 